\title{
A New Design of Two-Fingered Catheter Operating Robot for Vascular Interventional Surgery
}

\author{
Cai Meng ${ }^{1 *}$, Shengnan Sun ${ }^{2}$, Shaoya Guan ${ }^{3}$, Yin Liu ${ }^{4}$ and Tianmiao Wang ${ }^{5}$ \\ ${ }^{1}$ Associate Professor, School of Astronautics, Beihang University, Haidian District, Beijing, 100191, \\ China \\ ${ }^{2}$ Master student, School of Mechanism, Beijing University of Science \& Technology, Haidian District, \\ Beijing, 100191, China \\ ${ }^{3} \mathrm{PhD}$ student, School of Mechanism Engineering and Automation, Beihang University, Haidian \\ District, Beijing, 100191, China \\ ${ }^{4}$ Professor, School of Mechanism, Beijing University of Science \& Technology, Haidian District, \\ Beijing, 100191, China \\ ${ }^{5}$ Professor, School of Mechanism, Engineering and Automation, Beihang University, Haidian \\ District, Beijing, 100191, China
}

Received: June 25, 2017; Accepted: : July 17, 2017; Published: July 27, 2017

*Corresponding author: Cai Meng, Associate Professor, School of Astronautics, Beihang University, Haidian District, Beijing, 100191, China, and visiting scholar at Lab. for Computational Sensing and Robotics, the Johns Hopkins Univeristy, Baltimore, MD, 22218, USA. E-mail: Tsai@buaa. edu.cn

\begin{abstract}
It is of great value to design and develop CoR (Catheter-operating Robot) that can substitute a physician to manipulate a catheter in robotic-assisted vascular surgery. In this paper, we designed a new two-fingered CoR to simulate the catheter manipulation by a surgeon with thumb and forefinger, where the rotation and twist motions of the robotic fingers can achieve the conventional push / pull and rotation operation of the catheter respectively, and the open/close motion can achieve the rapid replacement of the catheter or operator. With the novel 'parts reuse' design, as few parts as possible are employed to achieve the two-finger movement in the mechanism structure, which lays a foundation for the miniaturization of the CoR in future. This paper first introduces the design and development of the proposed CoR, and then develops a CoR prototype. The operation precision of the CoR is analyzed by a quantitative experiment, and then a vitro-experiment is carried out to evaluate the validity of CoR in the robot assisted VIS with a glass vascular model. The experimental results show that CoR has the advantages of flexible control and high positioning accuracy, which is convenient for catheter control.
\end{abstract}

Index Terms: CoR (Catheter-operating Robot); Mechanism design; Part-reuse; VIS (Vascular interventional surgery);

\section{Introduction}

VIS (Vascular Intervention Surgery) is manually performed under the guidance of DSA (Digital Subtraction Angiography). Surgeons operate catheter, a semi-rigid soft pipe with guide wire, to move inside vessels for treatment of vascular disease.
At present, the VIS has been widely used in the treatment of cardiovascular and cerebrovascular diseases. In conventional VIS, surgeons operate catheter manually in operating room, so there are some disadvantages [1-2]. Firstly, the surgeons are exposed to X-ray for long time, which are harmful to their health. Secondly, long time operation and heavy lead-protection suits, both make surgeons fatigue. Thirdly, some human factors, such as surgeons' skill and hands tremble, may affect the success of surgery. Introducing a robot to VIS is an effective solution to solve these problems.

RVIS (Robot-assisted VIS) has many advantages compared to conventional VIS [3-7]. First, surgeons could control the robot outside of the operating room by remote operation, which can avoid X-ray irradiation. Second, the image navigation system of VIR reconstructs a 3D vascular model from 2D images, so surgeons do not need to reconstruct 3D vasculature in their minds, which reduces the surgery complexity. Third, the robot can operate catheter accurately and steadily, which decreases human influence (such as the tremor resulting from hands) on operation quality.

CoR(Catheter-operating Robot) is an important part in RVIS which manipulates the catheter directly. Several CoRs for RVIS have been developed in recent years [7-18]. However, most of the existing CoRs are still in the development stage or test stage, far from being commercial product for clinical application.

By analyzing the existing CoRs, we find that for most of them the catheter is placed inside the device. Medical staffs have to spend time to guide the catheter through the device, which 
results in extra workload. In addition, it will bring potential danger to surgery, because surgeons cannot take the catheter out from the CoR rapidly to restore manual operation when the RVIS breaks down. Bian et al. have tried to solve this problem by employing dual-finger design, but their mechanism does not use 'part-reuse' design which result their CoR has many components and is bulky [17-18]. In practice, the CoR will be fixed at the top of a supporting manipulator to control catheter axial and rotational motions. Therefore, the structure of CoR should be light and small-sized to meet the medical environment as well.

Aimed at this problem, a novel two-fingered CoR is developed to simulate the operating catheter with thumb and forefinger of a surgeon. In our mechanism, coaxial two-way screws design and integration design of sector gear and sliding part are adopted. The coaxial two-way screws design is employed to obtain twist motion of two-fingers while the sector gears works as sliding groove. The mutual axis and bi-directional sector gears design is utilized to obtain the open/close (or release/clamping) motion of dual-fingers. And a driving motor is integrated in the finger body to rotate the fingertip, which is to obtain the push or pull operation of the catheter. We have discussed this in our previous work in a workshop, but will elaborate the mechanism design in this paper [19].

In the following, we addressed the custom mechanical design and the control system of the CoR at first, then quantification experiment and vitro-experiment with transparent glass vessel model are carried out to evaluate the accuracy and validity the proposed CoR.

\section{Mechanism Design of Catheter Operating Robot}

\section{Analysis of the design requirements}

During conventional VIS, the surgeon manually inserts the catheter into the blood vessels to reach the lesion location. The catheter is pushed to advance axially along the blood vessels. When the catheter suffers from resistance blocking advancing or advances to a bifurcation of vessels, surgeons should rotate the catheter to adjust the orientation of catheter. The function of CoR is to assist the surgeons to perform the catheterization. The mechanism design should meet the requirements of operating tasks, surgeon's intervention, and operating environments. At the same time, CoR as a surgery robot must ensure the system's safety and reliability.

On the whole, the design of CoR should meet the following requirements:

1)control catheter to realize the motion along the catheter's shaft (axial motion) and about the shaft (radial motion);

2)small-sized and compact structure ;

3)taking and putting catheter rapidly, in case the robot breaks down;

4)the system should be compatible with catheters with different sizes;

5)achieve highly precise positioning, to ensure the catheter can reach the lesion location precisely;
The CoR our group developed includes translation/rotation module and clamping/loosening module, which are used to push/pull and rotate the catheter and clamp/loosen catheter easily, respectively.

\section{Translation/rotation module design}

Two bionic fingers (one is positive, the other is passive) are designed to simulate the manual operation, which is just like surgeons operate the catheter with their thumb and forefinger in the conventional VIS. The axial motion and radial motion of catheter are driven by friction between the two bionic fingers, which is adopted the friction principle. The principle of pushing/ pulling catheter is shown in Figure1 (a). When the catheter is clamped by the two bionic fingers, the positive bionic finger is driven to rotate, and then the passive bionic finger rotates synchronously and reversely relative to the positive. Finally, realize the catheter for axial motion.

The way of rotating catheter is the same as the way surgeons twist the catheter manually. When the catheter is clamped, both of the bionic fingers are driven to do the relative translation motion up and down. Finally, rotation motion of the catheter is realized. The principle of rotating catheter is shown in Figure 1 (b).

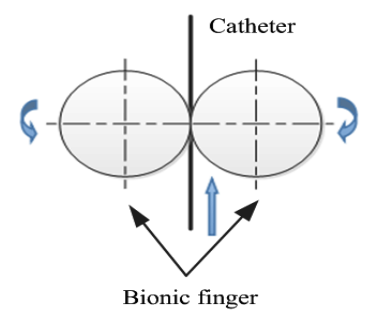

(a)

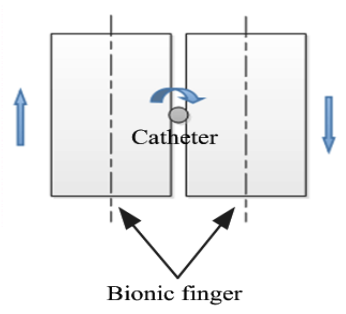

(b)
Figure 1: The principle of axial motion and radial motion of catheter: (a) axial motion, the two bionic fingers rotate synchronously and reversely relative to each other, the motion of the bionic fingers is mechanically transduced to a translation of the catheter via friction between the catheter and the bionic fingers, and (b) radial motion, the two bionic fingers are driven to do the relative translation motion up and down, the motion of the bionic fingers is mechanically transduced to a rotation of the catheter via friction between the catheter and the bionic fingers.

The rotation of positive bionic finger is driven by translation motor. Considering VIS has a high requirement for catheter's axial motion accuracy, we adopt translation motor to drive the positive bionic finger directly, which can avoid return error introduced by reducer. The catheter can be pulled or pushed continuously by the two bionic fingers rotating relatively, which improves the way that the surgeons operate the catheter manually.

Radial motion of catheter is driven by a rotation motor. In order to achieve the two bionic fingers' synchronous relative motion up and down, we need to transduce rotational motion of the rotation motor into linear. In mechanisms, there are multiple transmission mechanisms which can transform rotation motion into linear, such as crank-slider mechanism, cam mechanism, gear-rack mechanism, leadscrew transmission mechanism and the trolley mechanism. Considering saving space and ease of implementation, we choose leadscrew-nut mechanism as the 
transmission mechanism of CoR. Figure 2 shows two structure sketches designed for rotation module.

As shown in Figure 2 (a), two pairs of leadscrew-nut mechanism are symmetrically mounted, and the two leadscrews are fixed with two passive bevel gears respectively, by which the rotation motion of the active bevel gear can be converted to synchronous relative rotation of the two leadscrews. Limit the rotation of the nuts, so the rotation motion of the active bevel gear can be further converted into synchronous relative motion up and down of the two nuts.

As shown in Figure 2 (b), the leadscrew-nut pair is made up of a reverse leadscrew, which is a leadscrew with two-direction thread (the half of the leadscrew is right-handed thread and the other half is left-hand), a right-handed nut, and a left-handed. Limit the rotation of the nuts, so the rotation motion of the reverse leadscrew can be converted to synchronous relative translation motion up and down of the two nuts.

Compared to the structure as shown in Figure 2(a), the structure shown in Figure 2(b) is easier to ensure the assembling precision; in addition, this structure is simpler and more compact. So we finally adopt the structure shown in Figure 2(b).

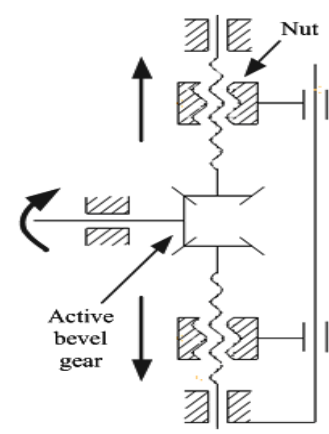

(a)

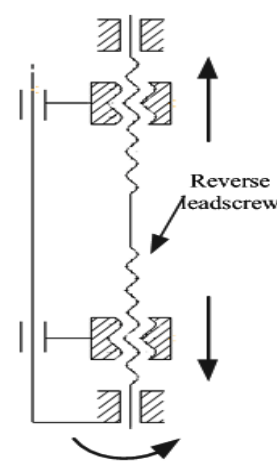

(b)
Figure 2: Structure sketch of rotation module: (a) the two pairs of leadscrew-nut mechanism are symmetrically mounted and the two leadscrews fixed with the two passive bevel gears respectively, limit the rotation of the nuts, so the rotation motion of the active bevel gear can be converted to synchronous relative motion up and down of the two nuts, (b) a reverse leadscrew (with two-direction thread) is adopted. When the reverse lead screw is driven to rotate, limit the rotation of the nuts, so the rotation motion of the reverse lead screw can be converted to synchronous relative translation motion up and down of the two nuts.

In order to ensure the transmission stable and no vibration, we choose synchronous belt mechanism as the transmission mechanism (Figure 3), rather than gear mechanism the most commonly adopted. A belt tensioner is designed for regular tension of the belt mechanism.

\section{Clamping/loosening module design}

The catheter is clamped by positive and passive bionic fingers. Clamping/loosening module is used to adjust the clamping distance between the fingers. So the CoR can drive different-sized

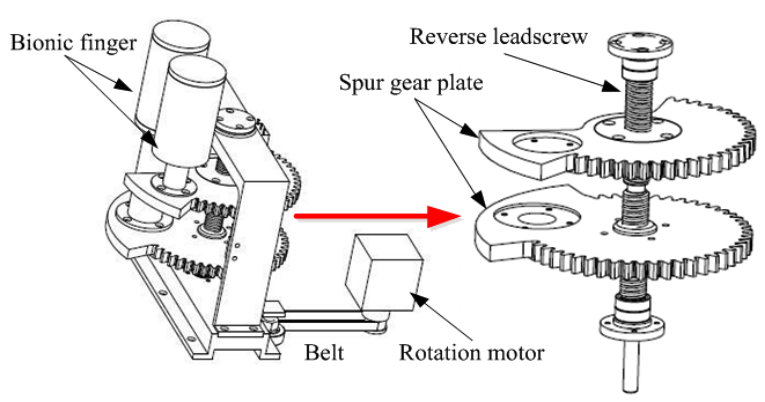

Figure 3: The 3D model of translation/rotation module..

catheters and realize taking and putting catheter easily. When the VIR breaks down, the catheter can be dismantled quickly and easily. In this case, surgeons handle the surgery manually.

The two bionic fingers are installed onto the moving parts which are fixed with the two nuts, respectively. So the two moving parts' rotation motion relatively about the leadscrew shaft is equivalent to adjusting the clamping distance between the two bionic fingers. Considering the nuts' degree of freedom of rotation needs to be limited, the clamping/loosening module had better be able to play the role of guide mechanism of the nuts.

The clamping/loosening module mainly adopts gear transmission mechanism (Figure 4). The section in wireframe of Figure 4 denotes the clamping/loosening module. Two passive bevel gears symmetrically arranged are fixed on two spur gears respectively. When the active bevel gear rotates, the two spur gears will rotate relatively. The two spur gears always keep meshing with two spur gear plates respectively, which are designed to hold the two bionic fingers, to transmit power and motion of the mechanical transmission (Figure 3). Finally, the motions of bionic fingers clamping or loosening catheter can be realized. By controlling the rotation angle and output torque of the clamping/loosening motor, the CoR can apply appropriate clamping force for catheters with different sizes.

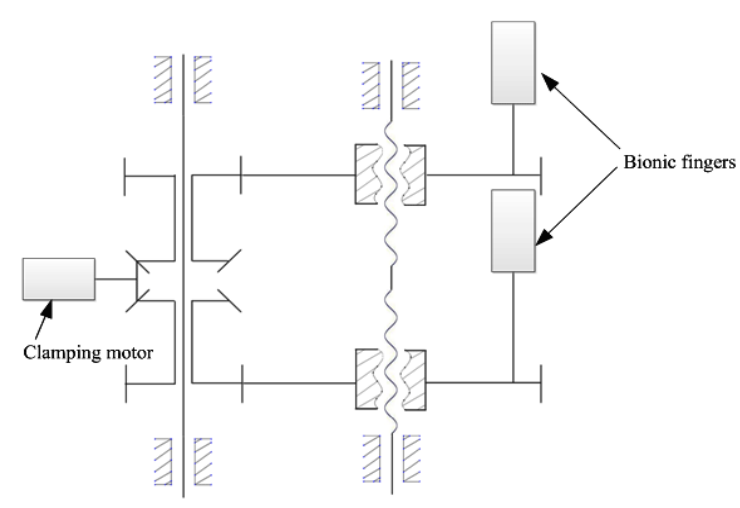

Figure 4:The active bevel gear meshes with two passive bevel gears, which are fixedly connected with two spur gears, and the two spur gears always keep meshing with two spur gear plates used to hold the two bionic fingers. 
When the clamping/loosening module play the role of guide mechanism, the spur gears keep unmoving under the static torque of the motor, so the rotation motion of the spur gear plates can be limited. Then, the translation/rotation module can work. The biggest advantage of the clamping/loosening module is that one module is both as clamping/loosening module and guide mechanism, which contributes to realize the miniaturization of structure.

\section{Realization of catheter operating robot}

Having finished the mechanical structure design of the CoR, this problem of motor selection for robot design need to be solved. The CoR is driven by three motors (Figure 5), which all have to start, stop, and change direction frequently; in addition, the motors are required to run in low velocity and must have sensitive reaction, high reliability, and high accuracy. Having analyzed the systematic population structure and various function indicators, we select stepping motors as the clamping/ loosening motor and rotation motor. Considering the translation motor need to be embedded in the active bionic finger, it should have a smaller-sized structure than the bionic finger, we choice a DC servo motor produced by the Swiss Maxon company as the translation motor. This motor is characterized by small volume, light weight and large output torque. In addition, the motor is equipped with a high precision encoder and a two-class gear reducer, the integration of which fully guarantee the reliability and precision of the whole drive solutions.

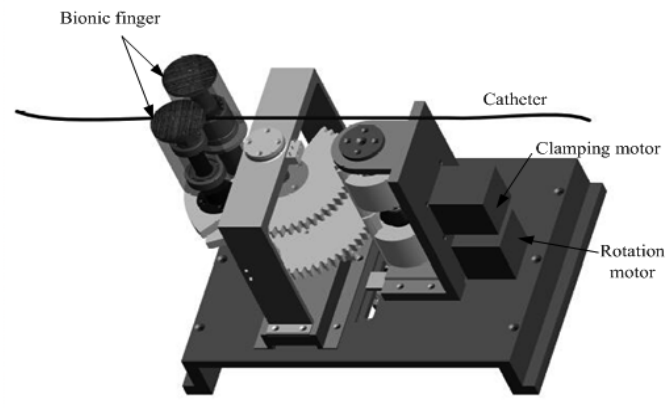

Figure 5: Structure of the CoR. The catheter is clamped by bionic fingers. Axial motion is implemented by translation motor driving active bionic finger directly. Radial motion is driven by rotation motor. The distance between the two bionic fingers is adjustable, so the robot can drive the catheters with different sizes. When the robot breaks down, the surgeon can take out the catheter quickly by control the clamping/ loosening module which is driven by a stepping motor, and switch to manual operation.

The prototype of CoR is adjusted after finely manufacturing and assembling. In order to increase the frictional force between the two bionic fingers, we cover a rubber sleeve on each bionic finger (Figure 6), which can prevent catheter slipping.

\section{Motion Control of CoR Control System}

In order to guarantee the function, safety, and reliability of VIS process, we develop an upper and lower classifying control system, which is a PC+PMAC (Programmable Multi Axis

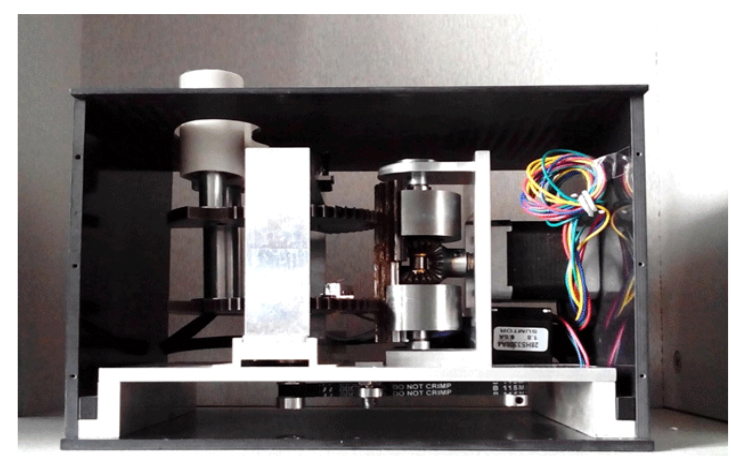

Figure 6: The prototype of CoR. We cover a rubber sleeve on each bionic finger to increases frictional force and prevent catheter slipping.

Controller) architecture system. As a host computer, PC realizes system management and human-computer interaction; PMAC as the lower machine achieves motors coordination control. This control system ensures the mechanical manipulator with stable performance and reliable control effect.

In the process of the operation, the surgeon sits in the monitoring room far from operating room, sending control instruction to the CoR. The control instruction is transferred effectively by PCI bus from PC to PMAC, which can further achieve servo control and motor coordination control. Finally, these instructions are translated into the motion of the catheter by the robot. The driver connected to the corresponding motor and the corresponding encoder installed on the end of motor form a close-loop controlling system, which could ensure that PC precisely controls the motor.

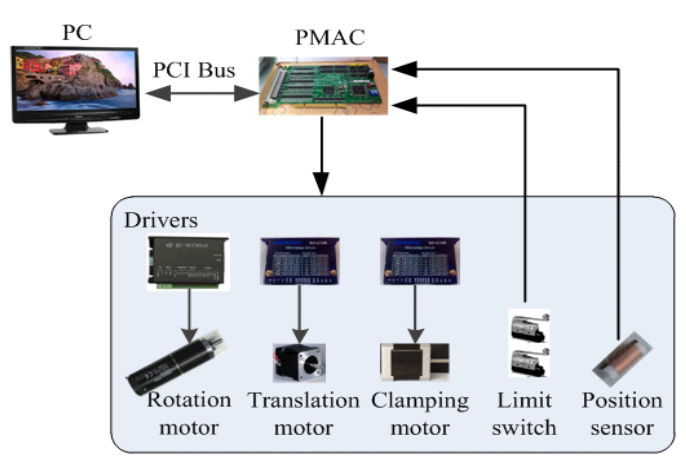

Figure 7: The three motors are controlled by a four-axis PMAC which communicates with the computer console.

\section{Motion model of catheter}

The output angle of translation motor or rotation motor can be got through the encoder installed on the end of each motor. According to the mechanical structure and dimension of the CoR, the relationships between the rotation angle of catheter and output angle of rotation motor, and axial displacement of catheter and output angle of translation motor, can be got. 


\section{A)Translation motion model of catheter}

The output angle of translation motor and the axial displacement of catheter are ( $\mathrm{rad})$ and ( $\mathrm{mm})$, respectively, which satisfy the following relationship:

$$
s=\frac{D}{2} \cdot \theta_{T}
$$

Where, $(\mathrm{mm})$ denotes the diameter of the bionic fingers.

\section{B)Rotation motion model of catheter}

The output angle of rotation motor and the displacement up or down of positive/passive bionic fingers is ( $\mathrm{rad}$ ) and ( $\mathrm{mm})$, which satisfy the following relationship:

$$
\frac{\theta_{R}}{2 \pi \cdot i}=\frac{y_{f i n}}{t}
$$

Where, tis the reverse leadscrew pitch, idenotes the transmission ratio of synchronous belt transmission mechanism. $y_{\text {fin }}$ and the rotation angle of catheter, $\alpha(\mathrm{rad})$, satisfied the following relationship:

$$
\frac{y_{f i n}}{\pi d}=\frac{\alpha}{2 \pi}
$$

Where, $\mathrm{d}(\mathrm{mm})$ denotes the diameter of catheter. According to (2) and (3), the relationship of rotation motor output angle, $\theta_{R}$ and catheter rotation angle, $\alpha$ can be obtained.

$$
\alpha=\frac{t}{\pi d \cdot i} \cdot \theta_{R}
$$

\section{Dynamics analysis of catheter}

In the process of catheterization, we must ensure that the catheter is clamped reliably by the two bionic fingers and does not slip in the mechanism when actuated. The clamping force is determined by the static torque of the clamping/loosening motor. So when going on the model selection of the clamping/loosening motor, the static torque of motor is a key consideration.

When the catheter is clamped reliably, the force of pushing or puling catheter completely depends on the output torque of the translation motor, the greater output torque of the translation motor, the greater pushing/puling force for catheter. As we all know, motor output torque is proportional to its current. So we can increase the force of pushing/puling catheter by increasing the translation motor current. In the same way, we can increase the force of rotating catheter by increasing the rotation motor current.

In the process of catheter moving in blood vessels, we can adjust the current of translation motor or rotation motor to adapt to the resistance value of different time, which will avoid catheter slipping caused by that the resistance acting on the catheter is greater than the motor driving force.

\section{Experiment}

In order to verify whether the CoR can meet the application requirements, some experiments are carried out to evaluate the accuracy and precision of the $\mathrm{CoR}$ and to validate the effectiveness of catheterization. Firstly, we conduct a series of experiments to characterize the accuracy and precision of the CoR in the axial direction and radial direction. And on this basis, an experiment is performed with a transparent glass vascular model to assess the usefulness of the CoR in robot-assisted VIS.

\section{Accuracy and Precision of Catheter Operating Robot}

\section{Evaluation in axial motion}

The accuracy and precision of catheter controlled by the CoR in axial direction is evaluated using a $4-\mathrm{F}$ (3.5-mm diameter) catheter, which is placed into a 4-mm diameter transparent plastic pipe, which was made to avoid measurement error introduced due to the elastic deformation of the catheter.

Treat the catheter as a rigid body; the pulse number sent to the translation motor, $P_{1}$ in correspondence with the theoretical displacement of the catheter can be calculated using (5), which is derived by (1):

$$
P \frac{360}{\pi \delta} s
$$

Where, $\delta_{1}\left({ }^{\circ}\right)$ expresses the rotation angle of translation motor when just one pulse sent to it.

In the prototype $\mathrm{CoR}$, when the theoretical displacement of catheter in axial direction, $\mathrm{s}$ is $1 \mathrm{~mm}$, the calculated value of $P_{1}$ is 205. The actual displacement of the catheter is measured using the Aurora sensor, a magnetic tracking sensor with five DOFs, embedded at the distal end of the catheter. By Aurora sensor, the spatial coordinates $(\mathrm{x}, \mathrm{y}$, and $\mathrm{z}$ ) of the distal end of the catheter can be feed backed. The actual displacement of the catheter $S_{a c t}$ is calculated by (6):

$$
\begin{aligned}
& s_{\text {act }}=\sqrt{\left(x_{2}-x_{1}\right)^{2}+\left(y_{2}-y_{1}\right)^{2}+\left(z_{2}-z_{1}\right)^{2}} \text { (6) } \\
& \text { Where }\left(x_{1}, y_{1}, z_{1}\right) \text { - - starting location; } \\
& \left(x_{2}, y_{2}, z_{2}\right) \text { - - arriving location. }
\end{aligned}
$$

To evaluate accuracy and precision, the catheter is advanced from 0 to $10 \mathrm{~mm}, 0$ to $20 \mathrm{~mm}, 0$ to $30 \mathrm{~mm}, 0$ to $40 \mathrm{~mm}$, and 0 to $50 \mathrm{~mm}$, ten times in succession, respectively.

Accuracy is evaluated by absolute error (between actual displacement of the catheter and the theoretical displacement), and then calculating the mean error of the absolute error. Axial precision is evaluated by calculating the standard deviation of the absolute error. 
The accuracy and precision of the CoR in the axial direction are listed in Table 1 . In the procedure of pushing catheter, the error range increases with the theoretical displacement, but the maximum of relative error (absolute error / theoretical displacement) is less than $\pm 5 \%$. During the surgery, when the catheter moves close to the bifurcation of vessels or the lesion location, the surgeon could improve the location accuracy by decreasing the single displacement of catheter.

\begin{tabular}{|c|c|c|}
\hline \multicolumn{2}{|c|}{ Table 1: Accuracy and Precision Of the Cor In Axial Direction } \\
\hline $\begin{array}{c}\text { Theoretical } \\
\text { displacement } \\
\text { (mm) }\end{array}$ & $\begin{array}{c}\text { PrEcision } \\
\text { (MM) }\end{array}$ & $\begin{array}{c}\text { Accuracy } \\
\text { (mm) }\end{array}$ \\
\hline 10 & \pm 0.12 & -0.04 \\
\hline 20 & \pm 0.26 & -0.09 \\
\hline 30 & \pm 0.50 & 0.17 \\
\hline 40 & \pm 1.00 & 0.30 \\
\hline 50 & \pm 1.10 & 0.34 \\
\hline
\end{tabular}

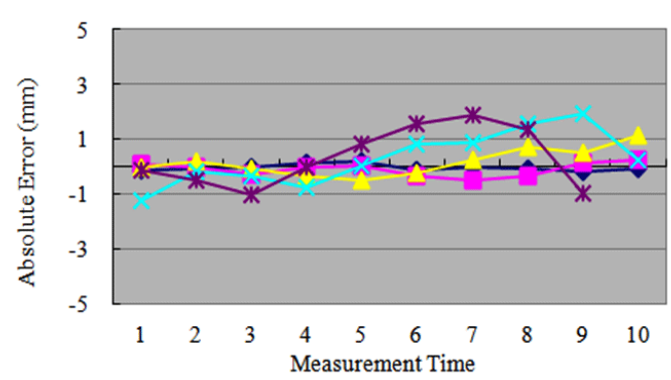

(a)

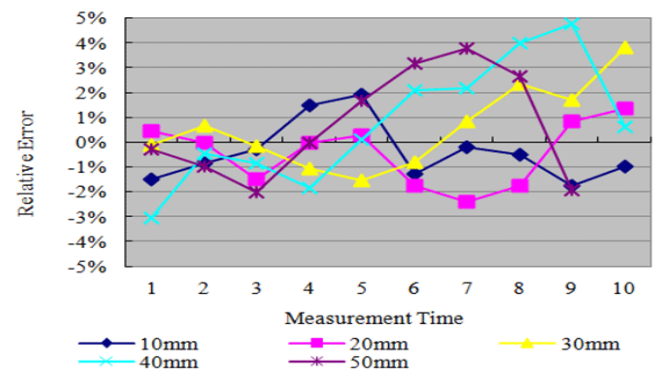

(b)

Figure $8: 10 \mathrm{~mm}, 20 \mathrm{~mm}, 30 \mathrm{~mm}, 40 \mathrm{~mm}$ and $50 \mathrm{~mm}$ are separately set as the theoretical displacement of the catheter, using a magnetic tracking sensor to measure the catheter's position, and then calculating the actual displacement of the catheter; thereby we can calculate (a) the absolute error, as well as (b) the relative error, i.e., divide absolute error by theoretical displacement of the catheter.

\section{B)Evaluation in radial motion}

To evaluate the accuracy and precision of radial motion, the $4-\mathrm{F}$ catheter is rotated by the CoR from $0^{\circ}$ to $360^{\circ}$ ten times, using a compass to measure the actual rotation angle of the catheter. The compass with a little hole at its origin is fixed on the CoR, and the catheter is placed through the hole. In order to facilitate to observe catheter's rotation angle, mark the catheter with a piece of colored tape (Figure 9).

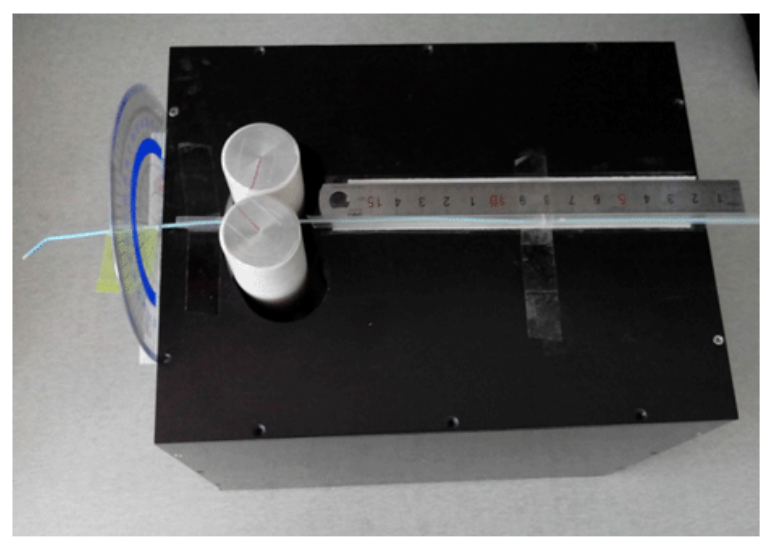

Figure 9: Catheter rotation accuracy test

The pulse number sent to the rotation motor $P_{2}$ can be calculated by (7), which is derived from (4).

$$
{ }^{2}-\delta_{2} \sim
$$

Where $\left({ }^{\circ}\right)$ expresses the rotation motor output angle when just one pulse is sent to it. In the prototype CoR, when $\alpha$ is $360^{\circ}$, $\mathrm{P}_{2}$ is 27360 . The constant pulse is sent to the rotation motor repeatedly to rotate the catheter, recording the radial position of the catheter measured by the compass at each trial, and then the absolute error between the actual rotation angle and $360^{\circ}$ can be got (Figure 10).

The accuracy (mean error) and precision (standard deviation) of the CoR in the radial direction are listed in Table 2.

\begin{tabular}{|c|c|c|}
\hline \multicolumn{2}{|c|}{ Table 2: Accuracy and Precision Of the Cor In Radial Direction } \\
\hline $\begin{array}{c}\text { Theoretical angle } \\
\mathbf{(}^{\circ} \text { ) }\end{array}$ & $\begin{array}{c}\text { PrEcISION } \\
\mathbf{(}^{\circ}\end{array}$ & $\begin{array}{c}\text { Accuracy } \\
\mathbf{(}^{\circ}\end{array}$ \\
\hline 360 & \pm 7.50 & 2.37 \\
\hline
\end{tabular}

\section{Transparent glass vascular model experiment}

This experiment is done with a transparent glass vascular model to assess the usefulness of the CoR in robot-assisted VIS (Figure 11).

In this experiment, the catheter can be operated by the CoR to enter different vascular branches under the remote control of human operator and image guidance feedback. This indicates that the proposed CoR is feasible to operator the catheter directly instead of the surgeon.

\section{Result and Discussion}

We initially test the accuracy and precision of the CoR. The relative error of catheter for axial motion is within $\pm 5 \%$ and for radial motion is within $-1 \%$ to $-3 \%$, which can meet the requirements of clinical VIS. The accuracy and precision of the CoR are listed in Table I and Table II, for the axial direction and radial direction, respectively. The accuracy and precision of the CoR are mainly affected by the machining error and assembly 


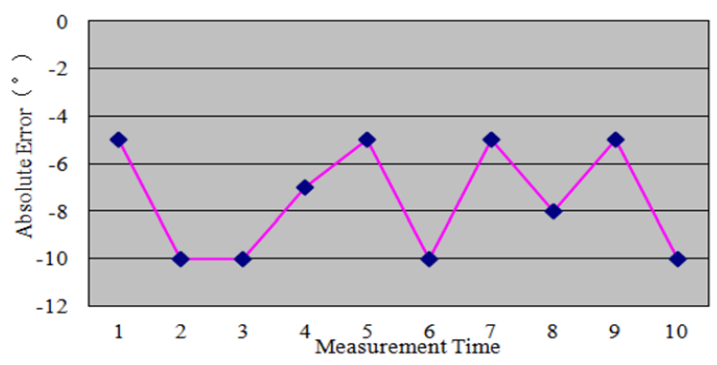

(a)

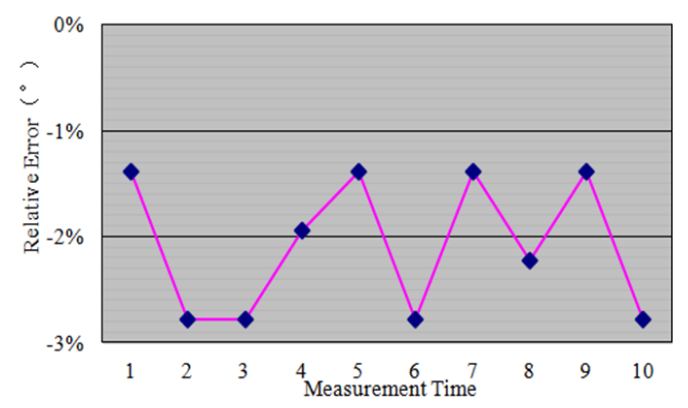

(b)

Figure 10: Repeatedly send 27360 pulses to the rotation motor, in theory, the catheter rotation angle should be $360^{\circ}$; use compass to measure the actual rotation angle, thereby we can calculate (a) the absolute error, as well as (b) the relative error, i.e., divide absolute error by $360^{\circ}$.

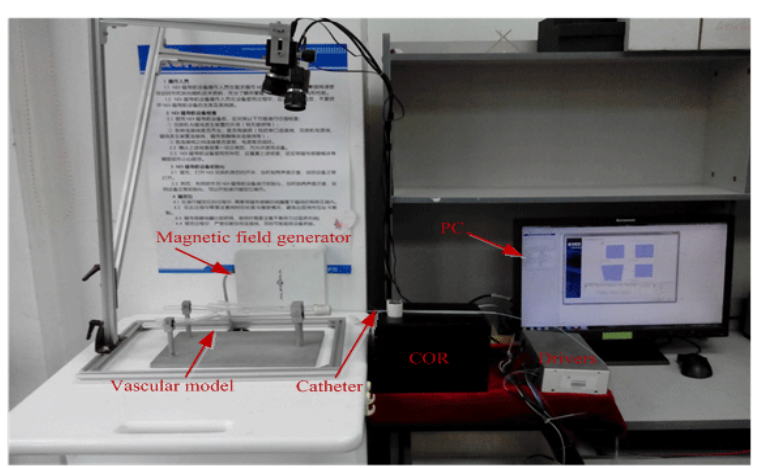

Figure 11: The transparent glass vascular model experiment platform: The motor drivers of the CoR connect to the PMAC, which communicates with the PC, so we can control the CoR by PC to pull, push or twist the catheter inside the vascular model. Image feedback is provided by an image navigation system developed by our group.

error; in addition the inevitable elastic deformation of catheter will cause measurement error. Besides, the measuring method itself exist measurement error. Especially for the radial motion, using a compass to measure the rotation angle of the catheter, which is an easy measuring method, but it will exist obvious measure errors. So the measuring method needs to be improved in the follow-up study.

Bases on the accuracy and precision test, the transparent glass vascular model experiment was carried out. The experiment showed that the catheter could be easily manipulated through the vascular branches. But the vascular model is a relatively simple environment; in the future, we should carry out more phantom experiments and animal experiment to assess the usefulness of the CoR in RVIS.

In the first prototype of the proposed CoR, the main object is to verify the feasibility of the design. Therefore, the weight and size are paid less attention. The total weight of the CoR prototype is $2.6 \mathrm{~kg}$ and the dimensions are $18 \times 12 \times 16\left[\mathrm{~cm} \rrbracket^{\wedge} 3\right.$. But they could be both decreased. The weight could be decreased because it was mostly made of aluminum alloy and steel due to fabrication. Titanium alloy and engineering plastics such as ABS could be adopted in most parts. The size could be decreased by optimizing the space layout, size design and choosing smaller drive motors.

\section{Conclusion}

In this paper, a novel two-fingered CoR is designed and a prototype is developed. The CoR can control the catheter axial and rotational motions by two bionic fingers working together, whose operation process is simple and easy to realize. The surgeon can manipulate the robot far away from the operating room, which protect surgeons from X-ray irradiation. The development of clamping/loosening module is convenient to mount and unmount the catheter. In addition, by controlling the rotation angle and output torque of the clamping/loosening motor, the CoR can apply appropriate clamping force for the catheters with different sizes. The design concept of "part reuse" is adopted, which contributes to reduce the quantity of the components in the structure. In order to meet all kinds of demand in the process of operation, the motions of push/pull catheter and rotating catheter are structurally decoupled, which means that the two kinds of motion can be performed either in sequence or simultaneously. the rotation angle and output torque of the clamping/loosening motor, the CoR can apply appropriate clamping force for the catheters with different sizes. The design concept of "part reuse" is adopted, which contributes to reduce the quantity of the components in the structure. In order to meet all kinds of demand in the process of operation, the motions of push/pull catheter and rotating catheter are structurally decoupled, which means that the two kinds of motion can be performed either in sequence or simultaneously.

Finally, the feasibility of the proposed TF-CoR is evaluated by a series of experiments. Experiment results show that the TF-CoR can meet the requirements of VIS.

\section{Acknowledgment}

This research was supported by the National Science Foundation of China under Grant No. 61533016 and partially by NIH under Grant No. EB015638.

\section{References}

1. Reddy VY, Neuzil P, Malchano ZJ, Vijaykumar R, Cury R, Abbara S, et al. View-synchronized robotic image-guided therapy for atrial fibrillation ablation: Experimental validation and clinical feasibility. Circulation. 2007;115(21):2705-2714. 
2. Patterson MS, Schotten J, Van Mieghem C, Kiemeneij F, Serruys PW. Magnetic Navigation in Percutaneous Coronary Intervention. Journal of Interventional Cardiology, 2006;19(6):558-565. DOI: 10.1111/j.1540-8183.2006.00202.x

3. Da L, Zhang D, Wang T. Overview of the vascular interventional robot. International Journal of Medical Robotics \& Computer Assisted Surgery. 2008;4(4):289-294. DOI: 10.1002/rcs.212

4. Thakur Y, Bax J S, Holdsworth DW, Drangova M. Design and performance evaluation of a remote catheter navigation system. IEEE Trans Biomed Eng. 2009;56(7):1901-1908. doi: 10.1109/TBME.2009.2017203

5. Antoniou GA, Riga CV, Mayer EK, Cheshire NJ, Bicknell CD. Clinical applications of robotic technology in vascular and endovascular surgery. J Vasc Surg. 2011;53(2):493-499. doi: 10.1016/j. jvs.2010.06.154

6. Li S, Shen J, Yan Y, Chen D. Overview of the vascular interventional surgery robot. Chinese journal of medical instrumentation. 2013;37(2):119-122.

7. Feng Z, Xie XL, Bian GB, Wei P, Hou ZG, Tan M, et al. Design and controlof a robotic system for minimally invasive vascular interventional surgery. Proceedings of the 32nd Chinese Control Conference. 2013:59765981.

8. Wang T, Zhang D, Liu D. Remote-controlled vascular interventional surgery robot. International Journal of Medical Robotics \& Computer Assisted Surgery. 2010;6(2):194-201.

9. Yili Fu, Anzhu Gao, Hao Liu, Guo S. The Master-slave Catheterization System for Positioning the Steerable Catheter. The International Journal of Mechatronics and Automation, 2011;1(3-4):143-152.

10. Guo S, Xiao N, Gao B. A Novel Robot-Assisted Catheter Surgery System with Force Feedback. Selected Topics in Micro/Nano-robotics for Biomedical Applications. 2013:175-190.

11. Ma X, Guo S, Xiao N, Guo J, Yoshida S, Tamiya T, et al. Development of a novel robot-assisted catheter system with force feedback. 2011 IEEE International Conference on Mechatronics and Automation. 2011:107-111. DOI: 10.1109/ICMA.2011.5985640
12.Fu Y, Gao A, Liu H, Li K, Liang Z. Development of a novel robotic catheter system for endovascular minimally invasive surgery. The 2011 IEEE/ ICME International Conference on Complex Medical Engineering. 2011:400-405. DOI: 10.1109/ICCME.2011.5876772

13.Xiao N, Guo S, Guo J, Xiao X, Tamiya T. Development of a kind of robotic catheter manipulation system. 2011 IEEE International Conference on Robotics and Biomimetics. 2011:32-37. DOI: 10.1109/ ROBIO.2011.6181258

14. Yang X, Wang H, Sun L, Yu H. Operation and Force Analysis of the Guide Wire in a Minimally Invasive Vascular Interventional Surgery Robot System. Chinese Journal of Mechanical Engineering, 2015;28(2):249257.

15.Srimathveeravalli G, Kesavadas T, Li X. Design and fabrication of a robotic mechanism for remote steering and positioning of interventional devices. International Journal of Medical Robotics \& Computer Assisted Surgery. 2010;6(2):160-170.

16.Payne CJ, Rafii-Tari H, Yang GZ. A force feedback system for endovascular catheterization. 2012 IEEE/RSJ International Conference on Intelligent Robots and Systems. 2012:1298-1304. DOI: 10.1109/IROS.2012.6386149

17. Bian GB, Xie XL, Feng ZQ, Hou ZG, Wei P, Cheng L, Tan M. 2013, August. An enhanced dual-finger robotic Hand for Catheter manipulating in vascular intervention: A preliminary study. 2013 IEEE International Conference on Information and Automation (ICIA). 2013:356-361. DOI: 10.1109/ICInfA.2013.6720323

18. Feng ZQ Bian GB, Xie XL, Hou ZG, Hao JL. Design and evaluation of a bio-inspired robotic hand for percutaneous coronary intervention. 2015 IEEE International Conference on Robotics and Automation (ICRA). 2015:5338-5343. DOI: 10.1109/ICRA.2015.7139944

19. Meng C, Guan S, Sun S, Liu Y, Wang T. A novel catheter operating robot for vascular interventional surgery. 2016 IEEE Workshop on Advanced Robotics and its Social Impacts (ARSO). 2016:304-309. DOI: 10.1109/ ARSO.2016.7736300 